\title{
Introduction : Dynamiques, tensions et vecteurs de professionnalisation des communicateurs
}

\author{
Dany Baillargeon, professeur, \\ Université de Sherbrooke, \\ dany.baillargeon@usherbrooke.ca \\ Marc D. David, professeur, \\ Université de Sherbrooke, \\ marc.d.david@usherbrooke.ca \\ François Lambotte, professeur, \\ Université catholique de Louvain, LASCO \\ francois.lambotte@uclouvain-mons.be
}

\begin{abstract}
Comme le mentionnent en préface les collègues Brulois et de la Broise, de nouvelles dynamiques - entre tous les acteurs concernés par le développement d'un corpus francophone de connaissances sur la professionnalisation des communicateurs - ne sauraient être lisibles si elles n'étaient d'abord visibles. L'objectif de cette revue est de permettre le partage des résultats de travaux scientifiques tant vers des chercheurs - nos collègues - que vers des praticiens concernés par la question de la professionnalisation et, de façon plus générale, intéressés par la mise en regard de leurs compétences par des connaissances. Il est aussi et réciproquement de permettre à ces praticiens de rendre compte de leur démarche réflexive à partir de l'analyse de leurs routines de travail, des enjeux éthiques, des us et coutumes de la fonction, des dilemmes, des décalages entre le dire et le faire, des jeux d'acteurs, en un mot de leurs pratiques dans la singularité de leur contexte de travail. L'enjeu est, en fin de compte, d'assurer un lien permanent entre fondamentaux théoriques et pratiques développées, entre réflexion et action.

Prenant des positions inspirantes appuyées sur les réalités des professionnels de la communication en même temps qu'il les renvoie à des analyses critiques, ce premier cahier du RESIPROC sur les dynamiques de professionnalisation, tente de faire ce rapprochement délicat entre pragmatisme et apport scientifique; entre volonté de nourrir la réflexion théorique tout en donnant une voix à ces praticiens en proie aux mêmes questionnements que les chercheurs : qu'est-ce qu'être un communicateur de métier?
\end{abstract}


Baillargeon et al. s'interrogent sur les dynamiques de la constitution d'une figure du professionnel soutenue par le discours sur le professionnel (ethos), les actions prises pour soutenir cette figure (praxis) et les objets en démontrant l'existence (artéfacts). Par des entretiens auprès de représentants actuels et passés de sept associations professionnelles belges, françaises et québécoises, les auteurs montrent que de nombreuses tensions participent à créer cette figure professionnelle. Promue par un idéal associatif, cette dernière est en effet ballottée entre une quête de professionnalisation déontique et stratégique, mais que les tâches quotidiennes phagocytent, tel que l'atteste l'«apparent débalancement entre les artéfacts normatifs et leur réelle performativité dans la praxis et dans l'ethos ».

Cette même idée de tensions, inhérentes à ce qui constitue le caractère professionnel du communicateur, on la retrouve dans le texte de Valérie Lépine sur le rapport à l'évaluation de l'efficacité des communications. Jouant un rôle de premier ordre dans la constitution de ce qui fait professionnel, l'évaluation de la performance communicationnelle ne va pas sans heurts et force le professionnel à désinstrumentaliser son expertise pour la recadrer réflexivement, sous peine de devenir un technicien de la performance. Ce qui n'est pas sans impact, selon Lépine, sur l'identité des professionnels et sur l'institutionnalisation, surtout scolaire, des professionnels de la communication.

Autre tension, cette fois sur fond de représentation sociale des professionnels de la communication interne, Brulois et Charpentier la lisent en regard du désir des professionnels de s'extraire de la fonction pour adopter une posture plus humaine, plus sociale du communicateur. Brulois et Charpentier, à la lumière des entrevues menées auprès des dircoms en communication interne, proposent de voir le professionnel autant dans la façon qu'il a de relayer l'information que dans la façon qu'il relie les gens. Toutefois, s'extraire de la fonction suppose pour le communicateur de soutenir la «violence de l'organisation» qui les conduisent à « conjuguer trois rythmes : celui de l'action, celui de l'adaptation de l'individu aux changements, celui de la réflexion, de l'analyse, de la mise à distance ». À l'instar de Valérie Lépine, les auteurs en viennent à questionner la formation universitaire dans ce qu'elle peut avoir de technicisante: " dans quelle mesure les référentiels universitaires de formation en communication pourraient-ils infléchir les référentiels métiers au lieu de seulement s'y ajuster? ».

Lambotte et al. y vont d'une réflexion sur les dispositifs pédagogiques entourant la notion de professionnalisation dans un contexte de formation universitaire. Dans leur article, les auteurs retracent une série d'enjeux entourant leur propre dispositif d'enseignement, mis en place autour d'un séminaire interactif en communication interne dans lequel des étudiants canadiens, français et belges ont collaboré pour produire un audit de communication interne fictif. Les auteurs concluent que cette approche qu'ils qualifient de «pédagogie active » est en adéquation avec les attentes et recommandations des associations professionnelles. 
À travers un appareillage textuel à caractère déontique, Patrice de la Broise interroge l'agentivité de codes professionnels, chartes et autres manifestes dans leur contribution performative à la professionnalisation des communicateurs. Transcendant le simple corpus de référence, l'auteur analyse la dynamique par laquelle des regroupements professionnels s'auto-régularisent à l'interne et à l'externe à travers leurs métiers et fonctions de référence. En ce sens, cette contribution constitue donc «une invitation à poursuivre, dans l'espace francophone, l'analyse dynamique d'une professionnalisation disputée par de multiples instances représentatives, sur différents modes rhétoriques et services aux professionnels ».

Finalement, Bernard Dagenais se questionne sur les tensions entre les professionnels des relations publiques confrontés à certaines pressions indues provenant de leurs mandants. En ce sens, l'auteur évoque « le fossé qui existe entre la notion d'éthique que prônent les professionnels des relations publiques et la dérive d'une certaine pratique des relations publiques où le professionnel se transforme en mercenaire ». Dès lors, le professionnel des relations publiques doit négocier un espace difficile entre l'intérêt public, sa conscience professionnelle, un idéal professionnel, un code déontologique et la réalité d'affaires. Pour éviter le "dérapage continuel de la profession des relations publiques», il faut, selon l'auteur, absolument que les associations professionnelles acceptent de livrer un combat sur la place publique contre les dérives du métier.

Cette première livraison des Cahiers du RESIPROC marque donc une étape importante d'un programme scientifique et éditorial dont nous souhaitons qu'il favorise la structuration et la dynamique d'un réseau international. Ce premier jalon en appellera d'autres et il s'agira donc d'aller plus loin, sachant que la régularité et la réciprocité des échanges recommandent de faciliter l'expression du plus grand nombre. À cela, nous ne doutons pas que les Cahiers du RESIPROC puissent y contribuer grandement. 